\title{
§ V From the Nineveh UGU treatise (BAM 480, 482)
}

The so-called UGU is the first treatise from the Nineveh Medical Encyclopaedia. Since, the general topic of the UGU Treatise is the human head, human eyes were also addressed.

\section{§ V.1 UGU 1 (BAM 480)}

Base Manuscript: $\quad$ NI (BAM 480), collated.

Museum Number: $\quad$ K 2354 + K $2412+$ K $2463+$ K $2491+$ K $3237+$ K $6447+$ K $7086+$ K $8356+$ K $8800+$ K $8842+$ K $9828+$ K $11868+$ K $13398+$ K $13399+$ Sm $637+$ Sm $1156+$ K 10428 [BAM 488] + K 16451 [AMT 3/4].

Origin and date: Nineveh, 7th century BC.

Type of tablet: Two-column, portrait-oriented tablet. Vertical column rulings done with a twisted thread, and horizontal dividing lines were drawn with an instrument. Round firing holes visible on the obverse but not to be seen on the reverse. Several lines on col iii $(46-47 ; 55)$ have been been intentionally erased after the tablet dried out (see also Finkel in Worthington 2005: 6).

Measurements: ca. $27,5 \times 16,7 \times 3,5 \mathrm{~cm}$.

Copy: BAM 480, BAM 488, AMT 3/4.

Photo/CDLI nr.: $\quad$ Plates 36-39/P365742.

Literature:

Köcher 1980a: xxiii-xxv. Finkel 1998: 81 fn. 10. Foster 2005: 992; Worthington 2005; 2007; Stol 2007a: 3ff.; Heeßel 2010: 48ff.; Scurlock 2014: 306ff.; Panayotov 2016a,b; Bácskay 2018a: 47ff.

NB: It is not necessary to provide parallels here, since this text is cross-referenced many times, see especially the works of Worthington 2005; 2007.

\section{Edition of the Relevant Prescriptions}

1 šumma amēlu muhhašu umma ukâl šer'ān tīb nakkapti iraššìma ìnāšu ișapparā

2 ìnāšu birrata ipita išita murdinna qūqāna ašâ

3 u dimta ittanaddâ šuššān qa sahlê buṭutta ina erî tețên tanappi
'If a man's cranium/brain holds heat (i.e. 'fever'), he has throbbing of the blood vessel of the temple, (and) his eyes blink, ${ }^{2}$ his eyes are blurred with dimness, cloudiness, blurring of the vision, murdinnu-'bramble', qūqānu'worm', ${ }^{3}$ and are constantly giving off tear(s): You grind up one third litre of 
4 rēška ukâl ina libbi šuššān qa teleqqe ina mê kasî talâš tugallab tașammidma UD.3.KAM lā ipatțar

\section{Alternative prescription 1}

5 šuššān qa sahlê šuššān qa qēm labti ina mê kasî talâš tugallab taṣammidma UD.3.KAM lā ipațtar

\section{Alternative prescription 2}

6 sahlê țēnēti kukra uhūla qarnānâ ina šikari talâš ašar šanîmma

\section{Alternative prescription 3}

7 murra emesalli malmališ taballal ina himèti tasâk īnī̌̌u teqqi

\section{Alternative prescription 4}

8 šuššān qa sahlê šuššān qa hașab tinūri ešeret šiqil hīqāti [...]

9 ina mê kasî talâš tugallab taṣammidma UD.3.KAM [lā ipatțar]

\section{Instruction for the patient and the healer \\ 10 arki nașmadāti annâti ešeret šiqil sahlê ša pî urși [...]}

sahlu-plant (and) bututta-terebinth with a pestle (and) you sift (it). ${ }^{4}$ (Now) it requires your attention, you take one third litre out of it (the mixture prepared above), knead (it) in the sap of a kasûplant. You shave (his head), bandage it (with it), and he shall not remove (the bandage) for three days.

\section{Alternative prescription 1}

${ }^{5}$ You knead one third litre of sahlû-plant (and) flour of roasted grain in the sap of a kasû-plant. You shave (his head), bandage it (with it), and he shall not remove (the bandage) for three days.

\section{Alternative prescription 2}

'You knead ground sahlû-plant, kukruaromatic (and) horned uhūlu-alkali in beer, ditto (= you shave his head, bandage it, and he shall not remove it for three days.)

\section{Alternative prescription 3}

${ }^{7}$ You mix in equal amounts bitter-plant (and) emesallu-saline solution. You pound (it) in ghee (and) daub his eyes.

\section{Alternative prescription 4}

${ }^{9}$ You knead ${ }^{8}$ one third litre of sahlû-plant, one third litre of sherd(s) of a tannouroven (and) ten shekels of diluted beer [...] ' in the sap of a kasû-plant. You shave (his head), bandage it, and [he shall not remove (the bandage)] for three days.

\section{Instruction for the patient and the healer}

${ }^{10}$ After these bandages (are applied): ${ }^{11}$ he (= the patient) shall collect into a loaf of bread ${ }^{10}$ ten shekels of sahlu-plant from the mouth of the mortar [which] ${ }^{11}$ was 
11 țābta țābātu lā šulputū ina akali issipma ikkal hamšat šiqil [...]

12 ina šikari rūšti tamahhaṣma išattī̌u [...]

\section{Instructions for the healer}

13 UD.1.KAM anzahha tasâk [...]

\section{Alternative prescription 1'}

14 ešeret šiqil inib kalbāni [...]

15 tahaššal tanappi ina mê kasî [talâš ...]

\section{Alternative prescription 2'}

16 šimbizidâ [...]

Alternative prescription 3'

$17[\mathrm{x}] \mathrm{x}[. .$.

18 tahaššal tanappi $[. .$.

Alternative prescription 4'

19 ešeret šiqil qēm kupsi tubbal [...]

\section{Alternative prescription 5'}

20 ešeret šiqil qēm kupsi tubbal [...]

21 UD.1.KAM irassu tașammid qaqqassu [...]

22 ana qaqqadī̌su tašappak ina bīti [ša tarāna išû tušeššebšu ...]

\section{Alternative prescription 6'}

23 ištēn šiqil rikibti arkabi mišil šiqil [šamma peșâ ...]

\section{Alternative prescription 7'}

24 šuššān qa zēr papparhî šuššān qa zēr lapti? [...] never crushed (together with) salt (or) vinegar, then he shall eat (it). ${ }^{12}$ You shall beat (it) in premium beer ${ }^{11}$ five shekels of [ground sahlû-plant .... ${ }^{12}$ and he drinks it, [... Bandages of sahlû-plant for the eyes.]

\section{Instructions for the healer}

${ }^{13}$ On day one you pound anzahhu-glass [...]

\section{Alternative prescription 1'}

${ }^{15}$ You crush and sift ${ }^{14}$ ten shekels fruit of kalbānu-plant, [...] ${ }^{15}$ [You knead (them)] in the sap of a kasû-plant. [...]

\section{Alternative prescription 2'}

${ }^{16}[$ You pound] šimbizidû-galena [...]

\section{Alternative prescription 3'}

${ }^{18}$ You crush and sift ${ }^{17}[\mathrm{x}] \mathrm{x}[\ldots]{ }^{18}[. .$.

\section{Alternative prescription 4'}

${ }^{19}$ You dry ten shekels of sesame husks powder [...]

\section{Alternative prescription 5,}

${ }^{20}$ You dry ten shekels of sesame husks powder [...] ${ }^{21}$ You bandage his chest for one day, (and) [...] his head ${ }^{22}$ You pour [ ${ }^{21} \mathrm{X}$ litre of sesame oil $]{ }^{22}$ over his head, (and) [let him sit] in a room [which has a canopy. You keep doing this for three days.]

\section{Alternative prescription 6'}

${ }^{23}$ [You pound] one shekel of bat guano, half shekel of [white plant and ...]

\section{Alternative prescription 7'}

${ }^{24}$ [You ...] one third litre seed(s) of papparhû-purslane, one third litre seed(s) of laptu-turnip' [...] 
Alternative prescription 8,

25 šuššān qa isqūqa ešeret šiqil arti ... [...]

Alternative prescription 9'

$26 z \bar{e} r$ sahlê [...]
Alternative prescription 8,

${ }^{25}$ [You ...] one third litre of coarse flour

(and) ten shekel(s) of ... leaves [...]

\section{Alternative prescription 9'}

${ }^{26}[$ You pound] seed(s) of sahlû-plant [...]

Although the beginning of i 27 is broken, structurally it seems that the following fragmentary lines are most probably alternative prescriptions as well.

\begin{tabular}{|c|c|}
\hline $\begin{array}{l}\text { Alternative prescription 10' } \\
28 \text { [...] talâš ašar šanîmma }\end{array}$ & $\begin{array}{l}\text { Alternative prescription 10' } \\
28[\ldots] \text { you knead (it), ditto. }\end{array}$ \\
\hline $\begin{array}{l}\text { Alternative prescription 11 } \\
29 \text { kalgukku [...] tasâk teqqi }\end{array}$ & $\begin{array}{l}\text { Alternative prescription 11' } \\
{ }^{29} \text { You pound kalgukku-red paste [...] (and) } \\
\text { daub (his eyes). }\end{array}$ \\
\hline Alternative prescription 12' & Alternative prescription 12' \\
\hline $\begin{array}{l}30 \text { šuššān qa arti titti }[. . . \text { tubbal ...] ina mê } \\
\text { kasî talâšs }\end{array}$ & $\begin{array}{l}{ }^{30}[\text { You dry] one third litre of fig leave(s) } \\
{[\ldots . .] \text { you knead (them) in the sap of a }}\end{array}$ \\
\hline $\begin{array}{l}31 \text { tatârma tubbal tahaššal [tanappi ... } \\
\text { tugallab] tașammidma ašar šanîmma }\end{array}$ & $\begin{array}{l}\text { kasû-plant, }{ }^{31} \text { You dry again (and) crush } \\
\text { [(and) sift ... you shave (his head)], you } \\
\text { bandage (his head) and ditto (= and } \\
\text { daub his eyes). }\end{array}$ \\
\hline Alternative prescription 13' & Alternative prescription 13' \\
\hline $\begin{array}{l}32 \text { šuššān qa būšāna šuššān qa x [...]-ma } \\
\text { ašar šanîmma }\end{array}$ & $\begin{array}{l}{ }^{32}[\text { You ....] one third litre of būšānn-plant, } \\
\text { one third litre of } \mathrm{x}[\text { [.............] and ditto (= } \\
\text { and daub his eyes). }\end{array}$ \\
\hline Alternative prescription 14' & Alternative prescription 14 \\
\hline 33 uhūla qaimānâ [...] teqqi & $\begin{array}{l}{ }^{33}[\text { You ...] horned uhülu-alkali, [...] you } \\
\text { daub (his eyes). }\end{array}$ \\
\hline Alternative prescription 15 ' & Alternative prescription 15, \\
\hline 34 šušs & ${ }^{34}[$ You ...] one third [...] and ditto. \\
\hline
\end{tabular}

The passage until line i 40 is broken off, but structurally it seems that the following fragmentary lines are most probably alternative prescriptions as well. 


\section{Alternative prescription 16'}

41 [... talâš] tugallab tașammidma ašar [šanîmma]

\section{Alternative prescription 17'}

42 [... tatârma] tubbal tahaššal tanappi ina mê kasî talâš tugallab tașammidma ašar [šanîmma]

43 [... ina lipî eșemti] arikti tasâk teqqi

\section{Alternative prescription 18'}

44 [... šuhta ina libbi lipi] sāqi șabiti tasâk teqqi

\section{Alternative prescription 19'}

45 [...... tahaššal tanappi ina mê kasî] talâš tugallab tașammidma UD.3.KAM lā ipațtar

\section{Alternative prescription 20'}

46 [... ina] šizbi [talâš tugallab tașammidma ašar šanîmma

\section{Alternative prescription 21'}

$47[. .$.$] x [ina ]$ ] dišip šadî šuhat hurāṣi tasâk teqqi

\section{Alternative prescription 22'}

48 [.... tahaššal] tanappi ina mê kasî talâš tugallab taṣammidma UD.3.KAM lā ipațtar

\section{Alternative prescription 23'}

49 [.... tahaššal] tanappi ina šuršummī šikari bašli talâš tugallab taṣammidma UD.3.KAM lā ipațtar

\section{Alternative prescription 16'}

${ }^{41}[. .$. you knead (it) ...], shave (his head), bandage it (with it) and ditto (daub (his eyes).

\section{Alternative prescription 17'}

${ }^{42}$ You [dry again], crush and sift [...]. You knead (it) in the sap of a kasû-plant. You shave (his head), bandage it and ditto (daub (his eyes). ${ }^{43}[. .$.$] You pound [... in$ the marrow] of a long [bone] (and) daub (his eyes).

\section{Alternative prescription 18'}

${ }^{44}[. .$.$] You pound [copper patina into fat of$ a] gazelle's thigh and daub (his eyes).

\section{Alternative prescription 19'}

[ ${ }^{45}$ You crush and sift ...] You knead (it) in [the sap of a kasû-plant]. You shave (his head), bandage it (with it), and he shall not remove (the bandage) for three days.

\section{Alternative prescription 20'}

${ }^{46}$ [... you knead (it) in milk]. You shave (his head), bandage it (with it), and ditto (= he shall not remove (the bandage) for three days).

\section{Alternative prescription 21'}

${ }^{47}$ You pound ... [in?] mountain honey, patina of gold, you daub his eyes.

\section{Alternative prescription 22'}

${ }^{48}$ [You crush] and sift [...] You knead (it) in the sap of a kasû-plant. You shave (his head), bandage it, and he shall not remove (the bandage) for three days.

\section{Alternative prescription 23'}

${ }^{49}$ [You crush and] sift [...]. You knead (it) in boiled beer dregs. You shave (his head), bandage it, and he shall not remove (the bandage) for three days. 
Alternative prescription 24'

$50[. .$.$] arti? dumuqšina tatabbal ina dišpi$ tasâk teqqi

\section{Alternative prescription 25'}

51 [... dumuqšina] tahaššal tanappi ina mê kasî talâš tugallab tașammidma UD.3.KAM lā ipațtar

\section{Alternative prescription 26'}

52 [qēm] labti? tahaššal tanappi ina mê kasî talâš tugallab tașammidma UD.3.KAM lā ipațtar

\section{Alternative prescription 27'}

53 rikibti arkabi ina dišpi tasâk teqqi

\section{Alternative prescription 28,}

54 šuššān qa arti marišmalê tahaššal tanappi ina mê kasî talâš tugallab tașammidma UD.3.KAM lā ipațtar

\section{Alternative prescription 29'}

55 gașsa bašla ina šaman sirdi talâš tugallab tașammidma ašar šanîmma

\section{Alternative prescription 30'}

56 hașab igulê ša kibša išû ina šamni šuhti tasâk teqqi
Alternative prescription 24'

${ }^{50}[. .$.$] ... you remove the best parts of the$ foilage?, pound (them) in honey, (and) daub (his eyes).

\section{Alternative prescription 25,}

${ }^{51}$ You crush and sift [... their best] parts. You knead (them) in the sap of a kasûplant. You shave (his head), bandage it, and he shall not remove (the bandage) for three days.

\section{Alternative prescription 26'}

${ }^{52}$ You crush and sift [flour] of roasted grain. You knead (them) in the sap of a kasû-plant. You shave (his head), bandage it, and he shall not remove (the bandage) for three days.

\section{Alternative prescription 27'}

${ }^{53}$ You pound bat guano in honey (and) daub (his eyes).

\section{Alternative prescription 28'}

${ }^{54} \mathrm{You}$ crush and sift one third litre of marišmalû-plant leaves. You knead (them) in the sap of a kasû-plant. You shave (and), bandage (his head), and he shall not remove (the bandage) for three days.

\section{Alternative prescription 29'}

${ }^{55}$ You knead boiled gypsum in olive oil. You shave (and) bandage (his head), and ditto (= he shall not remove the bandage for three days).

\section{Alternative prescription 30'}

${ }^{56}$ You pound a fungus-ridden fineointment potsherd in oil (and) copper patina (and) daub (his eyes). 


\section{Alternative prescription 31'}

57 supāla sahlê tahaššal tanappi ina šuršummi šikari rūšti talâš tugallab taṣammidma UD.3.KAM lā ipatțar

\section{Alternative prescription 32'}

58 haltappāna kalbāna tahaššal tanappi ina šuršummī šikari bašli talâš tugallab tașammidma UD.3.KAM lā ipațar

\section{Alternative prescription 33'}

59 burāša kukra baluhha sahlê uhūla qarnānâ temessi ina šizbi talâš tugallab tașammidma UD.3.KAM lā ipatțar

\section{Alternative prescription 34'}

60 šuhta ina dišpi tasâk teqqi

\section{Alternative prescription 35,}

61 šuššān qa sahlê šuššān qa billata [ ...] talâš tugallab tașammidma UD.3.KAM lā ipațtar

\section{Alternative prescription 36'}

62 šuššān qa sahlê [...] talâš tugallab tașammidma UD.3.KAM lā ipațtar

Alternative prescription 37'

63 lulâ anzahha [...] tasâk teqqi
Alternative prescription 31'

${ }^{57}$ You crush and sift supālu-juniper (and) sahlû-plant. You knead (them) in the dregs of premium beer. You shave (and), bandage (his head), and he shall not remove (the bandage) for three days.

\section{Alternative prescription 32'}

${ }^{58}$ You crush and sift haltappānu-plant (and) kalbānu-plant. You knead (them) in the dregs of boiled beer. You shave (and) bandage it (his head), and he shall not remove (the bandage) for three days.

\section{Alternative prescription 33'}

${ }^{59}$ You wash juniper, $k u k r u$-aromatic, baluhhu-aromatic, sahlu-plant (and) horned uhūlu-alkali, and knead (them) in milk. You shave (and) bandage it (his head), and he shall not remove (the bandage) for three days.

\section{Alternative prescription 34'}

${ }^{60}$ You pound copper patina in honey, (and) daub (his eys).

\section{Alternative prescription 35'}

${ }^{61}$ You knead one third litre of sahlû-plant, one third litre of beer mash, [... in ...]. You shave (and) bandage it (his head), and he shall not remove (the bandage) for three days.

\section{Alternative prescription 36'}

${ }^{62}$ You knead one third litre of sahlû-plant, [...] [in ...]. You shave (and), bandage (his head), and he shall not remove (the bandage) for three days.

\section{Alternative prescription 37'}

${ }^{63} Y o u$ pound lulû-antimony, anzahhuglass, [...], (and) daub (his eyes). 


\section{Alternative prescription 38'}

64 šuššān qa qēm hallūri [šuššān qa qēm kakkî ... talâš tugallab tașammidma] UD.3.KAM lā ipatțar

\section{Alternative prescription 39'}

65 kasâ taqallu tahaššal tanappi ina šuršummī šikari talâš tugallab tașammidma UD.3.KAM lā ipatțar

\section{Alternative prescription 40'}

66 zēr lišān kalbi arqūssu tuhassâ mēšu ana pursìti tașahhat adi ablat tašakkan

67 enūma ìtablu ina šamni šuhti tasâk teqqi

\section{Alternative prescription 41'}

68 šamma peșâ rikibti arkabi lipi kanakti șumlalâ zēr šurdunî kamūn bīni zēr maštakal

$69 z \hat{e}^{?}$ kalbi arti bīni emesalli nīnâ zibâ mala niš inīka teleqqe

70 tapâs ina šamni tasâk in̄išu ina uhūli qarnānî temessi adi dimtu parsu teqqi enūma taṣammidušu teqqi arkišu

71 [itqūr] abāri ul tapațtaršu mê šunê tušabšal ana urî tessip ina kakkabi tušbât ina šêri qaqqassu

72 tapaššaš mê šunê bašlūti ana qaqqadišu tuqarrar qaqqassu kunša pušikki tarakkas
Alternative prescription 38'

${ }^{64}$ [You knead] one third litre of chickpea powder, [one third litre of lentil powder ... You shave (and) bandage (his head)], and he shall not remove (the bandage) for three days.

\section{Alternative prescription 39'}

${ }^{65} Y o u$ roast kasû-plant. You crush and sift (it). You knead (it) in beer dregs. You shave (and) bandage (his head), and he shall not remove (the bandage) for three days.

\section{Alternative prescription 40'}

${ }^{66} Y$ ou squeeze out seed(s) of 'dog'stongue plant', while still green. You press its liquid into a pursitu-bowl. You leave (it) until it is dried out. ${ }^{67}$ When it has dried out you pound it in oil (and) copper patina (and) daub (his eyes).

\section{Alternative prescription 41'}

${ }^{69}$ You take as much as you feel are required of ${ }^{68}$ white plant, bat guano, kanaktu-aromatic pith, șumlalûaromatic, seed(s) of šurdunû-plant, 'tamarisk cumin', seed(s) of maštakalplant, ${ }^{69} \mathrm{dog}$ 's dung?, leaf of tamarisk, emesallu-saline solution, mint, (and) black cumin. ${ }^{70}$ You crush (and) pound (the drugs) in oil. You wash his eyes in horned uhülu-alkali - until the tear(s) stop - and you daub (his eyes). When you bandage his (head) you daub (his eyes). Afterwards ${ }^{71}$ you do not stop treating him with the lead spoon-salve. You boil sap of a šunû-tree (and) collect (it) into an urû-bowl, let it stay under a star (= stay overnight). In the morning ${ }^{72}$ you smear his head. You trickle the 
73 [šuššān] qa šamna ana qaqqadǐ̌u tašappak ina bīti ša tarāna išû tušeššebšu UD.3.KAM annâ tēteneppuš boiled sap of a šunû-tree onto his head (and) tie his head with braid of combed wool. ${ }^{73}$ You pour [one third] litre of sesame oil over his head, (and) let him sit in a room, which has a canopy. You keep doing this for three days.

1 NI i 1 DIŠ NA UGU-šú KÚM ú-kal SA ZI SAG.KI TUK-ma IGI.MIN-šú $i$-BÀR

2 NI i 2 IGI.MIN-šú bir-ra-ta $a_{5}$ i-pi-ta $a_{5} i$-ši-ta $a_{5}$ mur-din-na qù-qa-na a-šấ-a

3 NI i 3 ù ÉR ŠUB.ŠUB- $a$ 1/3 SìLA ZÀ.HI.LI bu-țu-ta ina $^{\text {na4 }}{ }^{\text {URR }}$ À̀RA-en SIM

4 NI i 4 SAG-ka ú-kal ina ŠÀ 1/3 SİLA TI-qé ina A GAZI ${ }^{\text {sar }}$ SILA $_{11}-a s ̌$ SAR- $a b$ LAL-ma UD.3.KAM NU DU 8

5 NI i 5 1/3 SÌLA sah-lé-e 1/3 SİLA Zİ ŠE.SA.A ina A GAZI ${ }^{\text {sar }}$ SILA $_{11}-a s ̌ ~ S A R-a b$ LAL-ma UD.3.KAM NU DU 8

6 NI i 6 sah-lé-e ÀRA-tì šmGÚR.GÚR NAGA.SI ina KAŠ SILA ${ }_{11}$-aš ${ }^{\ulcorner} \mathrm{KIMIN}^{\mathrm{s}}$

7 NI i $7 \quad$ šm SES $^{\text {mun }}$ eme-sal-lì mál-ma-liš HE.HE ina İ.NUN SÚD IGI. ${ }^{r}$ MIN-šú $\urcorner$ $t\left[e^{?}-q i\right]$

8 NI i 8 1/3 SÌLA ZÀ.HI.LI 1/3 SİLA ŠIKA IM.ŠU.RIN.NA 10 GÍN $h i-q a-t[i \ldots . . . . .$.

9 NI i 9 ina A GAZI ${ }^{\text {sar }}$ SILA11- $a$ š SAR- $a b$ LAL-ma $\ulcorner$ UD.3?.K[AM NU DU 8 ]

10 NI i 10 EGIR na-aṣ-ma-da-ti an-na-ti 10 GÍN ZÀ.HI.LI ša KA ‘ur $r^{\urcorner}-s ̣[i$................]

11 NI i 11 MUN A.GEŠTIN.NA NU TAG.TAG ina NINDA is-sìp-ma GU 75 G[ÍN .....]

12 NI i 12 ina KAŠ.SAG SİG-aṣ-ma NAG-šú [..................................................

13 NI i 13 UD.1.KÁM AN.ZAH SÚ[D

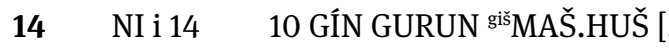

15 NI i 15 GAZ SIM ina A GAZ[I I ${ }^{\text {sar }}$ SILA $_{11}-a s ̌$ 


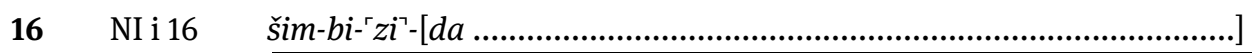

$17 \quad$ NI i $17 \quad[\mathrm{x}] \mathrm{x}[$.

18 NI i 18 GAZ SI[M

19 NI i $19 \quad 10$ GÍN Zİ ` DUH.ŠE.GIŠ.I’’ H[ÁD.DU

20 NI i $20 \quad 10$ GÍN Zİ DUH.ŠE.GIŠ.İ HÁD.D[U

$21 \quad$ NI i $21 \quad$ UD.1.KAM GABA-su LAL SAG.DU-s[u .

22 NI i 22 ana SAG.DU-šú DUB-ak ina 'É ${ }^{\top}[$.

(NI) ii 9 ana SAG.DU-šú DUB ina É šá ta-ra-na $a_{7}$ TUK-ú DÚR-šú UD.3.KAM an$n a_{7}$ DÜ.DÜ-uš

23 NI i $23 \quad 1$ GÍN U5.ARGAB ${ }^{\text {mušen }} 1 / 2$ GÍ[N Ú.BABBAR .........................................]

24 NI i 24 1/3 SÌLA NUMUN BABBAR.HI ${ }^{\text {sar }}$ 1/3 SÌLA NUMUN LU.[ÚB ${ }^{\text {sar? }}$...............]

$25 \quad$ NI i $25 \quad\ulcorner 1 / 3\urcorner$ SÌLA Zİ.KUM `10 GÍN PA 7 GI[Š .

26 NI i 26 [NUM]UN? sa[h?-lé-e

$27 \quad$ NI i $27 \quad$ Broken

$28 \quad$ NI i 28 SI]LA ${ }_{11}-a \check{S}^{\ulcorner} \mathrm{KIMIN}$

$29 \quad$ NI i 29

im KA[L.GUG. SÚ]D te-qí

$30 \quad$ NI i $30 \quad$ 1/3 SİLA PA giš PÈ [Š ... HÂD.A . in] $a$ A GAZI ${ }^{\text {sar }}$ SILA $_{11}-a s ̌$

31 NI i 31 GUR-ma HÁD.A G[AZ SIM SAR- $a] b$ LAL-ma KIMIN

32 NI i $32 \quad 1 / 3$ SÌLA úHAB 1/3 SİLA x [...........................................]- ${ }^{\ulcorner}$ma KIMIN ${ }^{\prime}$

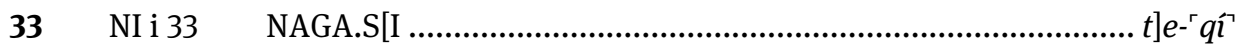


$34 \quad$ NI i 34

$35 \quad$ NI i 35 te-q]í

$36 \quad$ NI i 36 ] $x$

$37 \quad$ NI i 37 ] $x$

$38 \quad$ NI i $38 \quad$ Broken

39 NI i 39 Broken

$40 \quad$ NI i 40

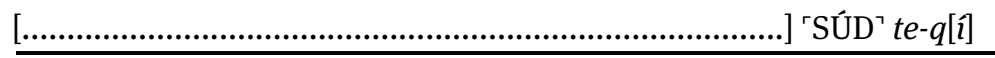

$41 \quad$ NI i 41 SILA $\left._{11}-a\right] \check{s}$ SAR- $a b$ LAL-ma K[IMIN]

$42 \quad$ NI i 42 GUR-ma HÁD.]A GAZ SIM ina A GAZI ${ }^{\text {rsarn }}$ SILA $_{11}-a s ̌ ~ S A R-a b$ LAL-ma KI[MIN]

$43 \quad$ NI i 43 ina İ.UDU GÌR.PAD.D]A GÍD.DA `SÚD? MAR

$44 \quad$ NI i 44 SAHAR.URUDU ina ŠÀ İ.UDU sa]-a-qí MAŠ. ‘DÀ SÚD te-qí

$45 \quad$ NI i 45

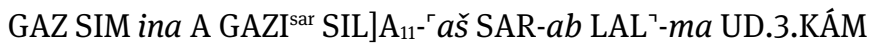
$\mathrm{NU} \mathrm{DU}_{8}$

$46 \quad$ NI i 46 ina] ${ }^{\ulcorner} \mathrm{GA}^{\urcorner}\left[\mathrm{SILA}_{11}-a s ̌ \mathrm{SA}\right] \mathrm{R}-a b$ LAL-ma KIMIN

$47 \quad$ NI i 47 .] x [ina ${ }^{~}$ LÀL ${ }^{7}$.KUR-i šu-hat KUG.GI SÚD MAR

$48 \quad$ NI i 48 GAZ SI]M ina A GAZI ${ }^{\text {sar }}$ SILA $_{11}-a \check{S}^{\ulcorner} \mathrm{SAR}^{\urcorner}-a b$ LAL-ma UD.3.KÁM $\mathrm{NU} \mathrm{DU}_{8}$

49 NI i 49 [............. GAZ SI]M 'ina ${ }^{7}$ šur-šum-mi KAŠ ŠEG 6. GÁ rSILA $\left.{ }_{11}-a s ̌\right\urcorner ~ S A R-a b$ LAL-ma UD.3.KÁM NU DU 8 
$50 \quad$ NI i 50 $\mathrm{P}] \mathrm{A} ? ? d u-m u q-\check{i}$-na ta-tab-bal ‘ina? LÀL SÚD te-qí

$51 \quad$ NI i 51

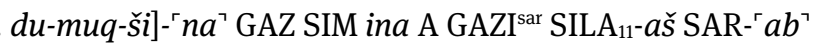
LAL-ma UD.3.KÁM NU D $\left[\mathrm{U}_{8}\right]$

52 NI i 52 [Zİ ŠE.SA]. ${ }^{\wedge} \mathrm{A}^{?\urcorner}$ GAZ SIM ina A GAZI ${ }^{\text {sar }}$ SILA $_{11}-a s ̌$ SAR- ${ }^{\circ} a b^{\urcorner}$LAL-ma UD.3.KÁM ${ }^{\ulcorner} \mathrm{NU}^{\mathrm{DU}} \mathrm{DU}^{\urcorner}$

$53 \mathrm{NI}$ i $53 \quad \mathrm{r}_{5}{ }^{\urcorner} \cdot\left[\mathrm{ARGAB}^{\mathrm{mu}}\right]^{\mathrm{s} \text { en }}$ ina $\mathrm{LÀL}{ }^{\ulcorner} \mathrm{SÚ} D$ te ${ }^{\urcorner}$-qí

54 NI i 54 1/3 SÌLA PA giš (OVER ERASURE) M[Á.ERI.I]Š.MÁ.RA GAZ SIM ina A GAZI ${ }^{\text {sar }}$ $\left[\mathrm{SILA}_{11}-a s ̌ \mathrm{~S}\right] \mathrm{AR}-\ulcorner a b\urcorner \mathrm{LA}[\mathrm{L}-m a]$ UD.3.KÂM NU DU 8

55 NI i 55 IM.BABBAR ba-aš-la 'ina İ’ sír-di SILA ${ }_{11}-a s ̌$ SAR-ab [L]AL-ma KIMIN

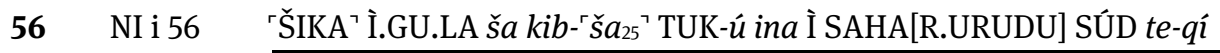

57 NI i 57 úZA.BA.LAM sah-lé-e ${ }^{\ulcorner}$GAZ SIM ${ }^{7}$ ina šur-šum-mi KAŠ.S[AG SILA S1- $_{11} a \check{s}$ S]AR- $a b$ LAL-ma UD.3.KÁM NU DU

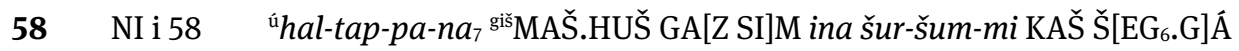
SILA $_{11}-a s ̌$ SAR- $a b$ LAL-ma UD.3.KÁM NU DU

59 NI i 59 šmLI šmGÚR.GÚR šmBULUH ZÀ.HI.L[I NA]GA.SI L[U]H-si ina GA SILA ${ }_{11}$ $a s ̌$ SAR- $a b$ LAL-ma UD.3.KÁM NU DU 8

60 NI i $60 \quad$ SAHAR.URUDU [ina L]ÀL SÚD te-qí

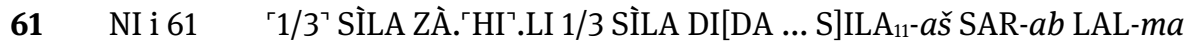
UD.3.KÁM NU DU

62 NI i 62 1/3 SìLA ZÀ.HI.L[I SI]LA ${ }_{11}-a \check{s}$ SAR- $a b$ LAL-ma UD.3.KÁM NU DU

63 NI i 63 KÙ.GAN AN.Z[AH? S]ÚD MAR 
64 NI i 64 1/3 SİLA Zİ G[Ú.GAL 1/3 SìLA Zİ GÚ.TUR ... SILA 11- $_{11} a \check{\text { SAR- }} a b$ LAL-m] $a$ UD.3.KÁM NU DU 8

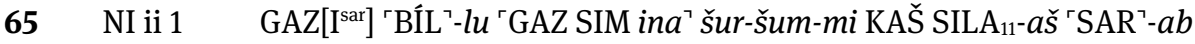
LAL-ma UD.3.KÁM NU DU

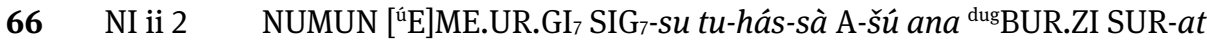
EN HÂD.DU GAR-an

67 NI ii 3 U[D-m]a i-tab-lu ina İ SAHAR.URUDU SÚD MAR

68 NI ii 4 'Ú.BABBAR? U ${ }_{5}$.ARGAB ${ }^{\text {mušen }}$ İ.UDU ${ }^{\text {šm }}$ GIG $^{\text {šm GAM.MA NUMUN }}{ }^{\text {úSI.SÁ }}$

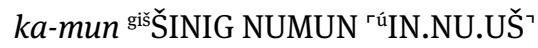

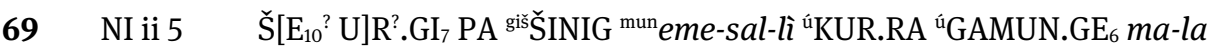
$n i-i s ̌$ IGI.MIN-ka ŠU.'TI ${ }^{r}$

70 NI ii 6 [t]a-pa-aṣ ina İ SÚD IGI.MIN-šú ina NAGA.SI LUH-si EN ÉR KUD-su teqî UD-ma LAL-šú te-qí EGIR-šú

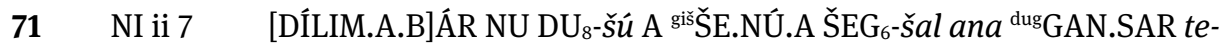
sip ina UL tuš-bat ina še-rì SAG.DU-su

72 NI ii 8 [Š]ÉŠ A gišŠE.NÚ.A ŠEG.GÁ ana SAG.DU-šú tu-qar-ra-âr SAG.DU-su

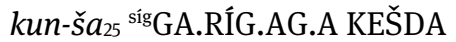

73 NI ii 9 [1/3? SÌL]A? İ.GIŠ ana SAG.DU-šú DUB ina É šá ta-ra-na 7 TUK-ú DÚRšú UD.3.KAM $a n-n a_{7}$ DÜ.DÙ-uš

74 [šumma amēlu] muhhašu? ìtenemmem ${ }^{74}$ [If man's] cranium/brain? is constantly gașșa uhūla qarnānâ barma kibrìt(a) eșemta uhūla qarnānâ napța u šaman nūni hot: ${ }^{75}$ You mix [together] ${ }^{74}$ gypsum, (multi-)coloured horned uhūlu-alkali, kibrìtu-sulphur, bone, horned uhūlualkali, naphtha and fish oil. ${ }^{75}$ You

75 [ištēniš] taballal ina pēmti ašāgi fumigate his head over charcoal of qaqqassu tuqattar ašāgu-acacia.

74 NI ii 10 [DIŠ NA? UG]U?-šú KÚM.KÚM-em IM.BABBAR NAGA.SI IN.GÙN kib-rit GÌR.PAD.DA NAGA.SI İ.HUL $u$ İ.KU 6 


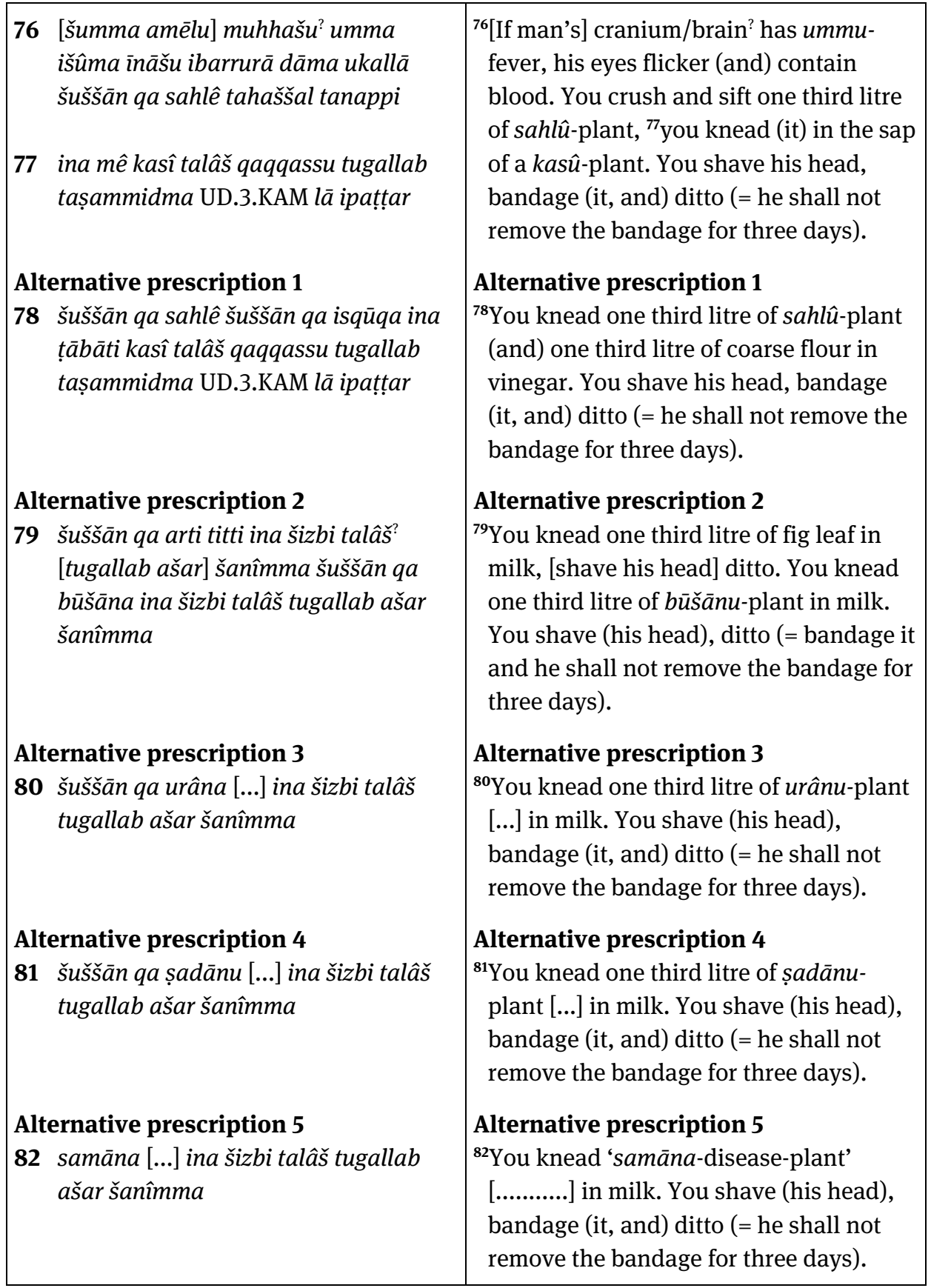


76 NI ii 12 [DIŠ NA? UG]U?-šú KÚM TUK-ma IGI.MIN-šú i-bar-ru-ra MÚD ú-kal-la 1/3 SİLA ZÀ.HI.LI GAZ SIM

77 NI ii $13 \quad$ 'ina $\mathrm{A}^{\urcorner}\left[\mathrm{GAZI}^{\mathrm{s}}\right]^{\text {ar }}$ SILA ${ }_{11}-a s ̌$ SAG.DU-su SAR- $a b$ LAL-ma UD.3.KÂM NU $\mathrm{DU}_{8}$

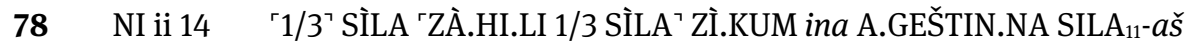
SAG.DU-su SAR- $a b$ LAL-ma UD.3.KÁM NU DU 8

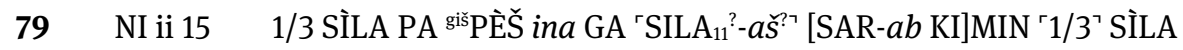
úHAB ina GA SILA H1- $a \check{S}$ SAR- $a b$ KIMIN

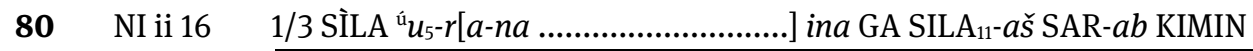

$81 \quad$ NI ii $17 \quad$ 1/3 SìLA ús

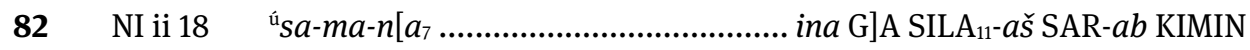

\section{§ V.2 UGU 2 (BAM 482)}

Base Manuscript: NX (BAM 482), collated.

Museum number: $\quad$ K $2392+2574+3430+3924+4091+10509+11744+16407(+) \mathrm{K}$ 2611

Origin and date: Nineveh, 7th century BC.

Type of tablet: Two-column, portrait-oriented tablet. Vertical column rulings done with an instrument, presumably a stylus. Several round firing holes visible on the reverse but not to be seen on the obverse.

Measurements: $\quad$ ca. 17,5 ×18,2×3,6 cm. (K 2392+); ca. 8,5×18,2×3,6 cm. (K 2611).

Copy: $\quad$ BAM 482; JMC 9: 47.

Photo/CDLI nr.: $\quad$ Plates 40-41/P365744.

Literatur:

Attia and Buisson 2003, additions in Attia and Buisson 2007: 47ff.;

Heeßel 2010: 50ff.

NB.

It is not necessary to provide parallels here since this text is cross-referenced many times, see especially the works of Attia and Buisson 2003, 2007. 


\section{Edition of the Relevant Prescriptions}

1 šumma [amēlu nakkapti] imittīšu iṣbassuma ìn imittišu dimta ukâl sahlê qēm labti

2 [pappas] buqli ištēniš taballal ina mê kasî tarabbak nakkaptašu tașammidma iballuṭ
${ }^{1}$ If a man's [right temple] afflicts him and his right eye contains tear(s): ${ }^{2} Y o u$ mix together ${ }^{1}$ sahlû-plant, flour of roasted grain, ${ }^{2}$ (and) malt [porridge]. You make an infusion from (them) in the sap of a kasû-plant. You bandage his (right) temple, then he should recover.

1 NX ii 20 DIŠ [NA SAG.KI ZAG-š] ' 'DAB-su-ma` IGI ZAG-šú ÉR ú-kal sah-lé-e Zİ ŠE.SA.A

2 NX ii 21 B[A.BA.ZA M]UNU 6 1-niš HE.HE ina A GAZI ${ }^{\text {sar }}$ tara-bak SAG.KI-šú LALma TI-út

3 šumma amēlu nakkapti šumēlišsu iṣbassuma īn šumēlišu dimta ukâl sahlê hašâ tahaššal tanappi

4 ina šikari bašli tarabbak nakkaptašu tașammidma iballut
3If a man's left temple afflicts him and his left eye contains tear(s): you crush and sift sahlû-plant and hašû-thyme. ${ }^{4} Y o u$ make an infusion from (them) in boiled beer. You bandage his (left) temple, then he should recover.

3 NX ii 22 'DIŠ NA SAG.KI' GÙB-šú DAB-su-ma IGI GÙB-šú ÉR ú-kal sah-lé-e úHAR.HAR GAZ 'SIM?

4 NX ii 23 ina KAŠ AL.ŠEG 6 .GÁ tara-bak SAG.KI-šú LAL-ma TI-`úṭ?

5 šumma amēlu nakkaptāšu șabtāšuma īnāšu dimta ukallā sahlê hašâ kasâ

6 qēm labti pappas buqli ištēniš ina kanni ina mê kasî tarabbak nakkaptǐ̌u takașșarma iballuṭ
${ }^{5}$ If both man's temples are afflicted and his both eyes contain tear(s): ${ }^{2}$ you make an infusion from sahlû-plant, hašû-thyme, kasû-plant ${ }^{6}$ flour of roasted grain (and) malt porridge in a kannu-jar, in the sap of the kasû-plant. You bind his temples, then he should recover.

5 NX ii 24 DIŠ NA SAG.KI.MIN-šú șab-ta-šú-ma? IGI.MIN-šú ÉR ú-kal-la sah-lé-e úHAR.HAR GAZI ${ }^{\mathrm{sar}}$

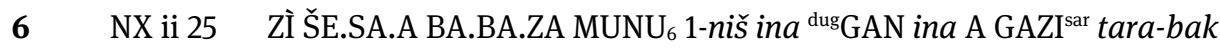
SAG.KI.MIN-šú KEŠDA-ma TI-uț 


\begin{tabular}{|c|c|c|}
\hline 7 & $\begin{array}{l}\text { šumma amēlu nakkapti imittīšu } \\
\text { iṣbassuma ìn imittǐšu dāma ukâl } \\
\text { sahlê hašâ gașṣa } \\
\text { dadāna qēm labti pappas buqli } \\
\text { ištēniš tahaššal tanappi ina țābāti } \\
\text { tarabbak ŠU.BI.AŠ.ÀM }\end{array}$ & $\begin{array}{l}\text { 7If a man's right temple afflicts him and } \\
\text { his right eye contains blood: }{ }^{8} \text { you } \\
\text { crush and sift together sahlû-plant, } \\
\text { hašû-thyme, gypsum, dadānu-acacia, } \\
\text { flour of roasted grain (and) malt } \\
\text { porridge. You make an infusion from } \\
\text { (them) in vinegar. Ditto (= you tie his } \\
\text { temple, then he should recover.) }\end{array}$ \\
\hline & \multicolumn{2}{|c|}{$\begin{array}{l}\text { DIŠ NA SAG.KI ZAG-šú DAB-su-ma IGI ZAG-šú MÚD ú-kal sah-lé-e } \\
\text { üHAR.HAR IM.BABBAR }\end{array}$} \\
\hline & \multicolumn{2}{|c|}{$\begin{array}{l}\text { gišKIŠI }{ }_{16 . H A B} \text { Zİ ŠE.SA.A BA.BA.ZA MUNU }{ }_{6} \text { 1-niš GAZ SIM ina } \\
\text { A.GEŠTIN.NA tara-bak ŠU.BI.AŠ.ÀM }\end{array}$} \\
\hline
\end{tabular}

9 [šumma] amēlu nakkapti šumēlīšu iṣbassuma īn šumēlī̌̌u dāma ukâl larda

10 šumutta ballukka ištēniš tahaššal tanappi ina [...] tarabbak šanîš
9[If] man's left temple afflicts him and his left eye contains blood: ${ }^{10}$ you crush and sift together ' 9 lardu-plant ${ }^{10}$ šumutta-plant (and) ballukkuaromatic, you make an infusion in [... and] ditto (= you tie his temple, then he should recover.)

9 NX ii 28 [DIŠ N]A 'SAG`'KI GÙB-šú DAB-su-ma IGI GÙB-šú MÚD ú-kal ['K]I.'KAL ' 'HI.RÍ.IN

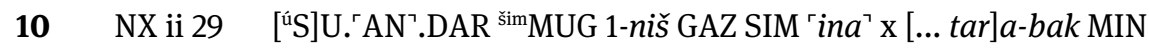

\section{BREAK OF UNKNOWN LENGTH!}

11’ šumma amēlu nakkapti imittī̌̌u ikkalšuma ìn imittǐ̌̌ naphat u dimta inaqqi šugidimmak šani Ištar

12' ana bulluțǐšu sīhu argānu barīrāta ištēn šiqil ru'tītu maštakal

13’ qan šalāli ina isqūqi taballal ina šikari tarabbak tașammid
1'If man's right temple hurt him and his right eye is inflamed and gushes tear(s): (The cause is) 'Hand of the Ghost', deputy of Ištar. ${ }^{12}$ In order to heal it: ${ }^{13}$ 'you mix ${ }^{12}$ 'sìhu-artemisia, argānu-plant, barīrātu-sagapenum', one shekel of ru'tītu-sulphur, maštakalplant ${ }^{13}$ '(and) šalālu-reed in coarse flour. You make an infusion from (them) in beer (and) bandage (his head and eyes). 


\begin{tabular}{|c|c|c|c|}
\hline $\begin{array}{l}\text { Alt } \\
\text { šur }\end{array}$ & $\begin{array}{l}\text { ernative p } \\
\text { nma ašar š } \\
\text { šuršummé }\end{array}$ & $\begin{array}{l}\text { šikari rūšti suluppi Dilmun } \\
\text { lipi kanakti ina himēti } \\
\text { i }\end{array}$ & $\begin{array}{l}\text { Alternative prescription } 1 \\
\text { If ditto (= in order to heal it): }{ }^{14} \text { you } \\
\text { pound }{ }^{13} \text { residue of garum (fish sauce), } \\
\text { residue of strong vinegar, }{ }^{14} \text { 'dregs of } \\
\text { premium beer, Dilmun date(s) (and) } \\
\text { one shekel of kanaktu-aromatic pith in } \\
\text { ghee, (and) daub (his eyes). }\end{array}$ \\
\hline & NX ii 62' & \multicolumn{2}{|c|}{$\begin{array}{l}\text { DIŠ NA SAG.KI ZAG-šú GU } \text { - }_{7} \text { Šú-ma IGI ZAG-šú nap-hat ù ÉR BAL-qí } \\
\text { ŠU.GIDIM.MA šá-ni }{ }^{\mathrm{d}} \check{I}_{8} \text {-tár }\end{array}$} \\
\hline & NX ii 63’ & \multicolumn{2}{|c|}{ 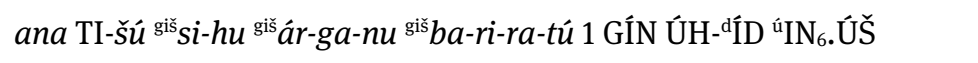 } \\
\hline & NX ii 64' & \multicolumn{2}{|c|}{$\begin{array}{l}\text { GI.ŠUL.HI ina Zİ.KUM HE.HE ina KAŠ tara-bak LAL : DIŠ KIMIN šur- } \\
\text { šum-mi ši-iq-qí šur-šum-me A.GEŠTIN.NA KALA.GA }\end{array}$} \\
\hline & NX ii 65’ & \multicolumn{2}{|c|}{$\begin{array}{l}\text { šur-šum-mi KAŠ.SAG ZÚ.LUM.MA Dilmun }{ }^{\mathrm{ki}} 1 \text { GÍN İ.UDU šm }{ }^{\text {sim }} \text { ina } \\
\text { İ.NUN SÚD te-qi }\end{array}$} \\
\hline
\end{tabular}

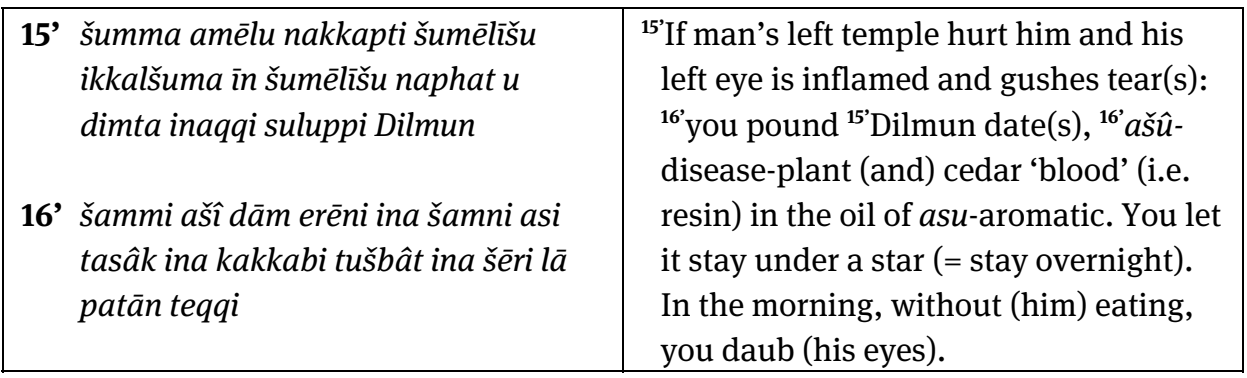
15’ NX iii 1 DIŠ NA SAG.KI GÙB-šú GU GU $_{7}$ '̌́ $\left.-{ }^{-} m a\right\urcorner$ IGI 150-šú nap-hat $u$ ÉR BAL-qí ZÚ.LUM.MA Dilmun ${ }^{\mathrm{ki}}$

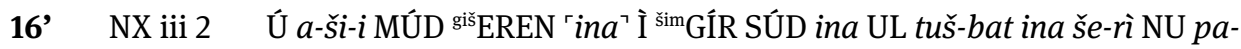
tan MAR

\begin{tabular}{|c|c|c|}
\hline $17^{\prime}$ & $\begin{array}{l}\text { šumma ašar šanîmma gașși Purattu } \\
\text { qēm buqli qēm hallūri qēm kakkî qēm } \\
\text { kasî } \\
\text { tahaššal tanappi ina mê kasî } \\
\text { tarabbak nakkaptašu ìňu tașammid }\end{array}$ & $\begin{array}{l}\text { 17'If ditto: you crush and sift Euphrates' } \\
\text { gypsum, malt flour, chickpea powder, } \\
\text { lentil powder (and) powder of a kasû- } \\
\text { plant. }{ }^{18} \text { 'You make an infusion (from } \\
\text { them) in the sap of a kasû-plant, (and) } \\
\text { bandage his temple (and) eye. }\end{array}$ \\
\hline & 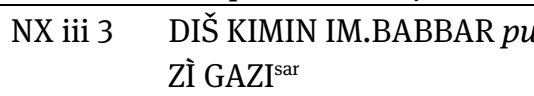 & \\
\hline
\end{tabular}


18' NX iii 4 GAZ SIM ina A GAZI ${ }^{\text {sar }}$ tara-bak SAG.KI-šú ERASURE IGI-šú LAL

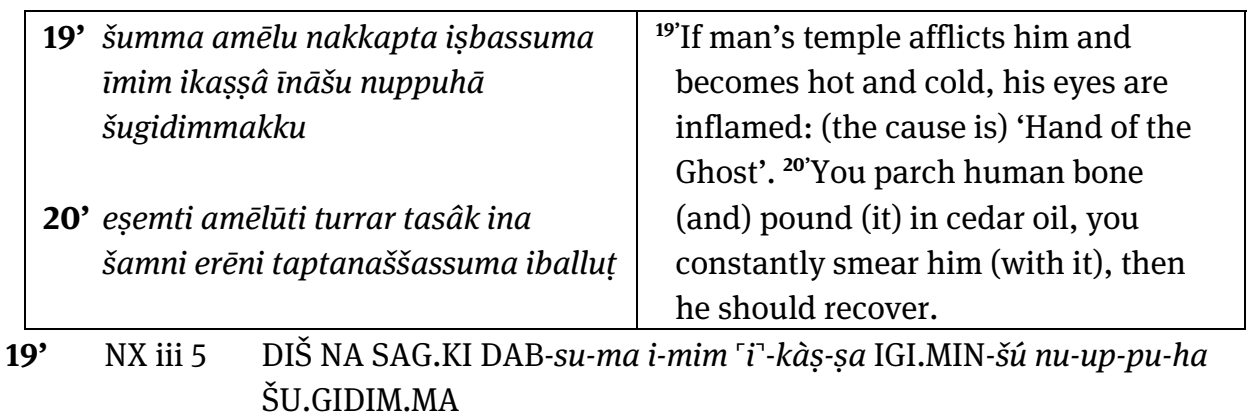

20’ NX iii 6 GÌR.PAD.DU NAM.LÚ.U $18 . L U$ tur-ár SÚD ina İ giš EREN EŠ.MEŠ-su-ma TI

\section{§ V.3 NY (AMT 25/8)}

Manuscript: $\quad$ NY (AMT 25/8), collated.

Museum number: British Museum, K 16449.

Origin and date: Nineveh, 7th century BC.

Type of tablet: The flake comes from the middle part of a possibly two-column tablet and might duplicate BAM 494 ii 53-57 (UGU 5?), but the lines do not seem to fit.

Measurements: $\quad$ ca. $3,1 \times 2,4 \times 0,5 \mathrm{~cm}$.

Copy: $\quad$ AMT 25/8

Photo/CDLI nr.: $\quad$ Plate 41/P402032.

Literature: $\quad$ Bácskay and Simkó 2017: ms. M.

\begin{tabular}{|l|l|l|}
\hline Ll. 1ff.' & parallels & BAM 494 ii 52ff.' \\
\hline
\end{tabular}

\section{Edition}

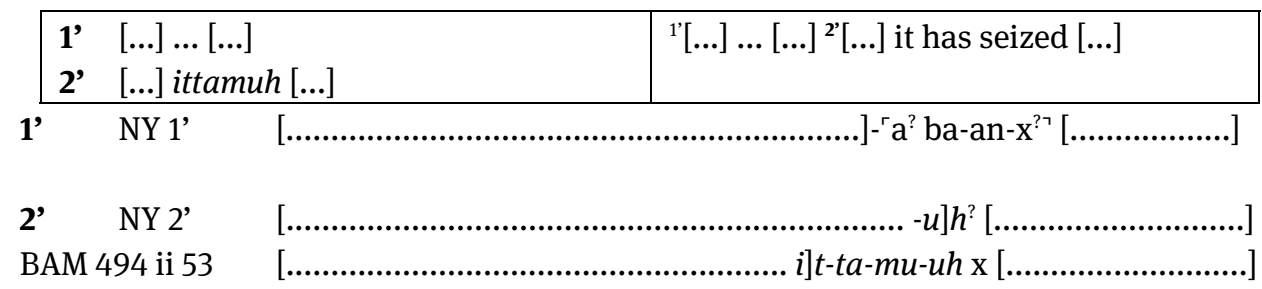




\begin{tabular}{|c|c|c|}
\hline $\begin{array}{l}3^{\prime} \\
\text { 4' }\end{array}$ & $\begin{array}{l}{[. . .] \text { ašâ ... [...] }} \\
{[\ldots] \text { zēr ašli ina pēmti tuqattaršuma }} \\
\text { ašâ innassah }\end{array}$ & $\begin{array}{l}{ }^{3}[\text { If man's eyes are ..........(and)] blurred } \\
{[(\ldots)]:{ }^{4} \text { you fumigate him with }{ }^{3}[(. . .)]} \\
4^{\prime}[\ldots . .] \text { (and) seed(s) of rush over } \\
\text { charcoal, and the } a \text { šû-disease will be } \\
\text { removed }\end{array}$ \\
\hline & \multirow{2}{*}{\multicolumn{2}{|c|}{ 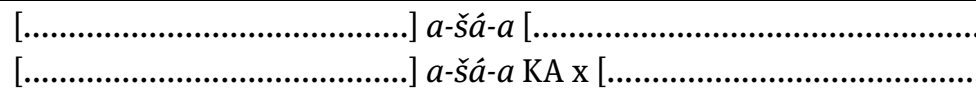 }} \\
\hline $\mathrm{BAM}$ & & \\
\hline
\end{tabular}

4’ NY 4' [...] x NUMUN (OVER ERASURE OF Ú) úaš-lì ina NE SAR-šú-ma M[AŠ ................]

BAM 494 ii $55 \quad$ [...................................... $a]$ š-lì ina NE SAR-šú-ma MAŠ.TAB.BA Z[I]

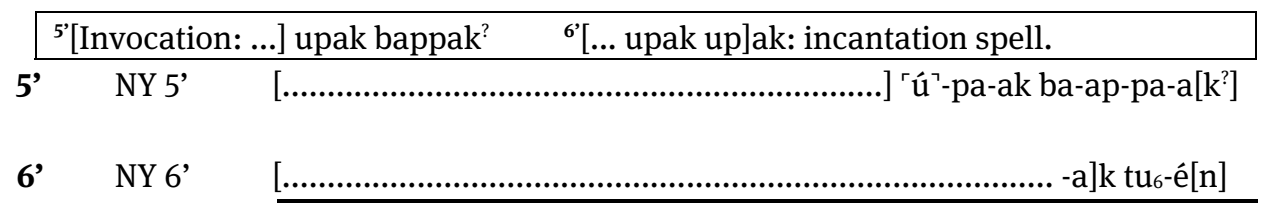

BAM 494 ii 56

.] x pa ú-pa-ak ú-pa-ak túb-é[n]

\begin{tabular}{|c|c|}
\hline $\begin{array}{l}\text { 7’ [...] dudubi mê nurmî [...] } \\
\text { 7a' [...] ana pî̌š tašappakma ina'eš }\end{array}$ & $\begin{array}{l}{ }^{7}[\ldots] \text { this medical application: }\left({ }^{8}[\ldots] \text { you }\right. \\
\text { pour) }{ }^{7} \text { pomegranate sap }[. . .] \text { into his } \\
\text { mouth }{ }^{8} \text { and then he should get better. }\end{array}$ \\
\hline NY 7’ & 'ÚR'.M[A ... \\
\hline BAM 494 ii 57 & 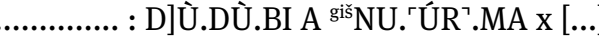 \\
\hline
\end{tabular}

7a' BAM 494 ii 58 .] ana KA-šú ta-šap-pa[k-ma in]a-[eš]

\section{§ V.4 NZ (AMT 5/3)}

Manuscript:

Museum Number:

Origin and date:

Type of tablet:

Measurments:

Copy:

Photo/CDLI nr.:

Literature:
NZ (AMT 5/3), collated.

K 10655 + K 10926 .

Nineveh, 7th century BC.

Fragment of a two-column tablet, containing typical prescriptions for the head. It is presumably to be placed somewhere in the Nineveh UGU treatise.

ca. $7,3 \times 5,5 \times 1,5 \mathrm{~cm}$.

AMT 5/3.

Plate 41/P398808.

Borger 1967: 529; Köcher 1980a: xxiv; Stol 1991/92: 58 note 102; Worthington 2005: 6. 


\section{Edition}

\begin{tabular}{|c|c|c|}
\hline & $\begin{array}{l}{[\ldots] \text { teleqqe }} \\
{[\ldots . . \text { ina] šamni tasâk }} \\
{[\ldots] \text { lā ipparras }}\end{array}$ & $\begin{array}{l}{ }^{\prime}[\ldots] \text { you take }{ }^{2}[\ldots], \text { (and) pound [in] } \\
\text { sesame oil } \\
3^{\prime}[\ldots] \text { (but the disease) cannot be } \\
\text { removed. }\end{array}$ \\
\hline 1' & {$[\ldots \ldots \ldots . . . .$.} & 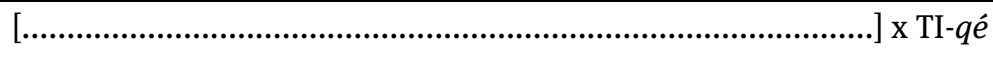 \\
\hline 2' & {$[\ldots \ldots \ldots . . . .$.} & [ \\
\hline 3' & {$[\ldots \ldots \ldots . . .$.} & {[} \\
\hline
\end{tabular}

\begin{tabular}{|c|c|c|}
\hline $\begin{array}{l}4^{\prime} \\
5 \\
6 \\
6^{\prime} \\
7\end{array}$ & $\begin{array}{l}{[\ldots \text { innāšu }] \text { dāma? ukallā }} \\
{[\ldots . .] \text { talâš tașammid }} \\
{[\ldots . .] \text { talâš ašar šanîmma }} \\
\text { [...] talâš ašar šanîmma }\end{array}$ & $\begin{array}{l}{ }^{4}\left[\ldots \text { his eyes] contain blood. }{ }^{5}[. . .] \text { you }\right. \\
\text { knead }[. . .] \text { (and) bandage. }{ }^{6}[\ldots] \text { you } \\
\text { knead }[. . .] \text { (and) ditto. }{ }^{7}[. . .] \text { you knead } \\
{[\ldots] \text { (and) ditto. }}\end{array}$ \\
\hline ' & NZ i 4' & ... IGI.MIN-šú MÚ]D? ú-kal-la \\
\hline 5' & 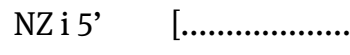 & SI]LA ${ }_{11}-a s ̌ \mathrm{LAL}$ \\
\hline $6^{\prime}$ & {$[\ldots \ldots \ldots \ldots \ldots . . . . .$.} & ................................... SILA \\
\hline 7' & 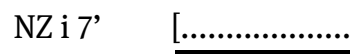 & .............................. X SILA11-aš KIMIN \\
\hline
\end{tabular}

\begin{tabular}{|c|c|c|c|}
\hline $\begin{array}{l}8^{\prime} \\
9^{\prime} \\
10^{\prime}\end{array}$ & $\begin{array}{l}{[\ldots] \text { innāš̀ }} \\
{[\ldots] \text { burāa }} \\
{[\ldots] \text { ina ̌̌ }} \\
\text { iballuṭ }\end{array}$ & $\begin{array}{l}\text { barrurā } \\
\text { șumlalâ baluhha } \\
\text { bi talâš tașammidma }\end{array}$ & $\begin{array}{l}\text { 8' }^{\prime} \text {...] (and) his eyes flicker. }\left[{ }^{10} \text { 'You }\right. \\
\text { pound? ....], }{ }^{\prime} \text { 'juniper, șumlalû- } \\
\text { aromatic, baluhhu-aromatic', }{ }^{10} \text { 'you } \\
\text { knead in milk [...] bandage (his eyes), } \\
\text { then he should recover. }\end{array}$ \\
\hline & NZ i 8 ' & \multicolumn{2}{|c|}{ 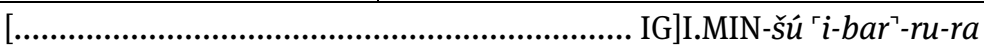 } \\
\hline 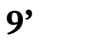 & NZ i 9 ' & \multicolumn{2}{|c|}{ 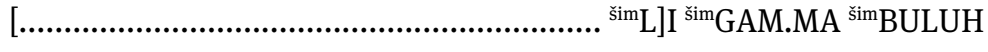 } \\
\hline 0' & NZ i 10’ & \multicolumn{2}{|c|}{ 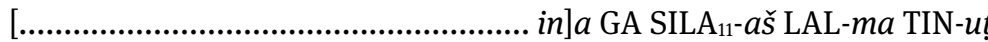 } \\
\hline
\end{tabular}

\begin{tabular}{|c|c|}
\hline $\begin{array}{l}\text { 11' }[. . .] \text { ina UD.1.KAM ša Nisanni } \\
\text { 12' [... țurri?] nabāsi tațammi }\end{array}$ & $\begin{array}{l}{ }^{11}[. . .] \text { on the first day of the month } \\
\text { Nisannu }{ }^{12}[\ldots] \text { you spin [a cord] of red } \\
\text { wool }\end{array}$ \\
\hline
\end{tabular}




\section{BREAK OF UNKNOWN LENGTH!}

\begin{tabular}{|c|c|}
\hline 13’ idra x [...] & 13’idru-saltpeter [...] \\
\hline
\end{tabular}

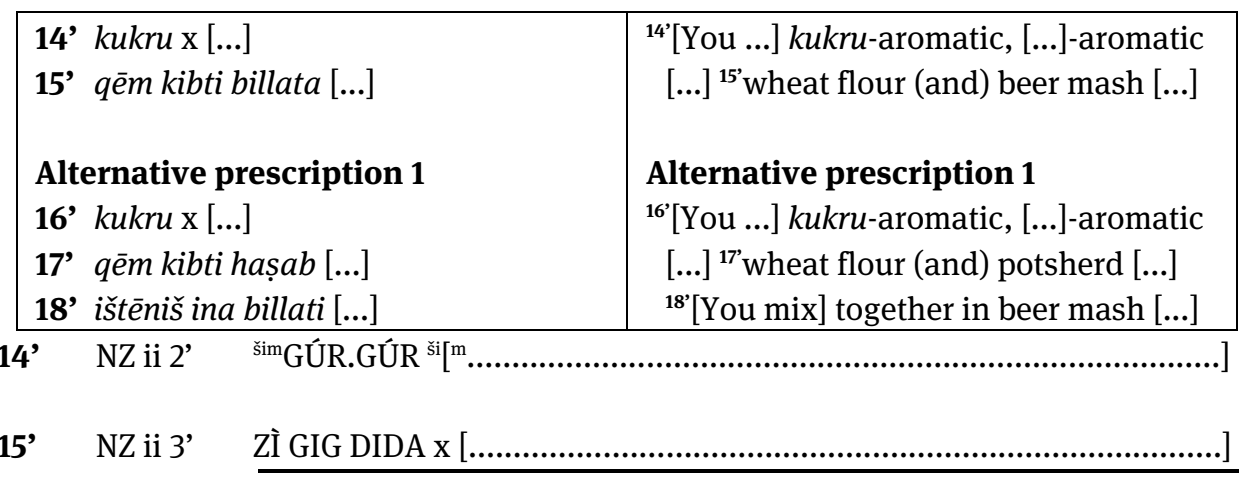

16. NZ ii 4’ šmGÚR.GÚR ${ }^{\text {sii }[m . .}$

17’ NZ ii 5, Zİ GIG ŠI[KA?

18' NZ ii 6’ 1-niš ina DID[A

\begin{tabular}{|c|c|c|c|}
\hline \multicolumn{3}{|c|}{ 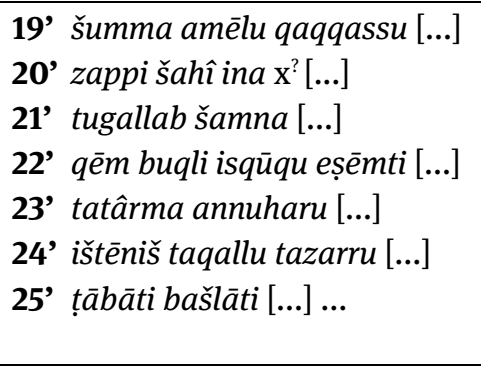 } & $\begin{array}{l}{ }^{19^{\prime}} \text { If man's head }[. . .]^{20^{\prime}}[\mathrm{You} . . .] \text { pig's } \\
\text { bristle in ... [and .... }]^{21^{2}} \text { You shave (his } \\
\text { head) [and ...] sesame oil [...] }{ }^{22^{\prime}} \text { malt } \\
\text { flour, coarse flour, bone of [...] }{ }^{23^{\prime}} \text { You } \\
\text { do ... again and }{ }^{24} \text { you roast } \\
\left.{ }^{23^{\prime}} \text { annuharu-mineral [...] }\right]^{24^{\prime}} \text { (and) spatter } \\
\text { (his eyes/head) [.... }{ }^{25^{\prime}} \text { boiled vinegar } \\
{[\ldots] \text {... }}\end{array}$ \\
\hline & NZ ii 7 & \multicolumn{2}{|c|}{ 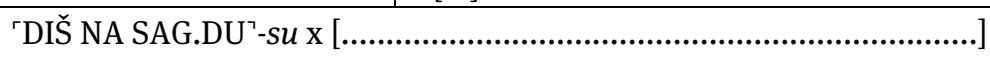 } \\
\hline & NZ ii 8 ' & \multicolumn{2}{|c|}{ 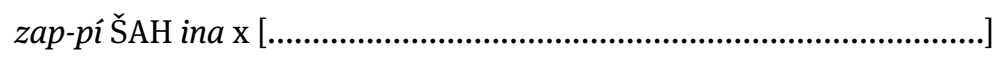 } \\
\hline & NZ ii 9’ & \multicolumn{2}{|c|}{ 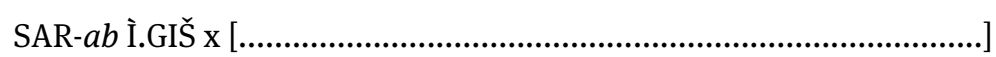 } \\
\hline & NZ ii 10 ' & \multicolumn{2}{|c|}{ 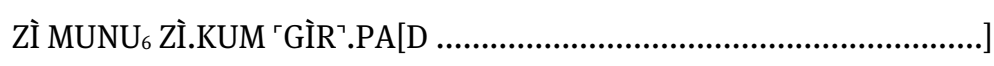 } \\
\hline
\end{tabular}


23' NZ ii 11' GUR-ma an-nu-ha-[ru ]

24’ NZ ii 12’ 1-niš BIL-lu MAR x [...

25’ NZ ii 13’ A.GEŠTIN.NA ŠEG ${ }_{6} \cdot G\left[\hat{A}^{?}\right.$

26’ NZ ii 14’ x x x x [

\section{§ V.5 NZa (AMT 13/5 + 14/5)}

Manuscript:

NZa (AMT 13/5 + 14/5), collated.

Museum Number: K 2974.

Origin and date: Nineveh, 7th century BC.

Type of tablet: $\quad$ Corner of possibly two-column tablet, which belongs to the treatise UGU 3, duplicating K 2516 (+) 2566+ i, bottom.

Measurements: ca. $7,7 \times 7 \times 3 \mathrm{~cm}$.

Copy: AMT 13/5 + AMT 14/5.

Photo/CDLI nr.: $\quad$ Plate 41/P394756.

Literature: Thomspon 1937b: 29 (no. 8), 32; Borger 1976: 529 and 236, 102/1 etc.; Scurlock 2006: 165f. (nos. 98 and 108-109, 116), 224 (com. ii 2f.), 681.

\section{Edition of the Relevant Prescription}

\begin{tabular}{|c|c|c|}
\hline $8^{\prime}$ & 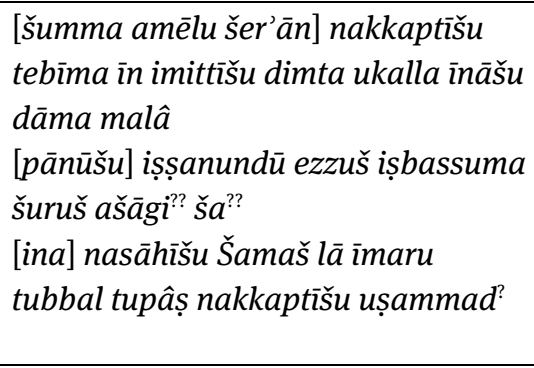 & $\begin{array}{l}\text { '[If man's] temple veins throb and his } \\
\text { right eye contains tear(s), his both } \\
\text { eyes are full of blood '’he has a vertigo } \\
\text { (and) his anger? afflicts him:you dry } \\
\text { and crush root of } a \text { šāgu-acacia', which } \\
\text { 10'has not seen the sun when it was } \\
\text { torn out, (and) he bandages both his } \\
\text { temples (with it). }\end{array}$ \\
\hline & \multicolumn{2}{|c|}{$\begin{array}{l}\text { [DIŠ NA SA SA]G.KI-šú ZI-ma IGI ZAG-šú ÉR ú-kal-la IGI.MIN-šú MÚD } \\
\text { DIRI- } a\end{array}$} \\
\hline & \multicolumn{2}{|c|}{ [IGI.MEŠ-šú] ri’-șa-nun-du e-zu-uš DAB-su SUHUŠ KI[ŠI ${ }_{16}$ š] $a^{?}$} \\
\hline & \multicolumn{2}{|c|}{ 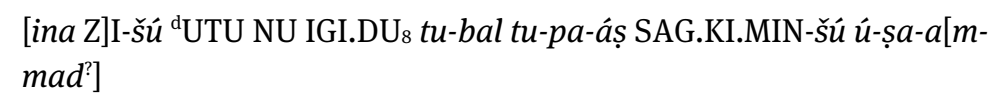 } \\
\hline
\end{tabular}

